\title{
Racial/Ethnic Disparities in Delaying or Not Receiving Medical Care During the COVID-19 Pandemic
}

J Gen Intern Med 37(5):1341-3 DOI: $10.1007 / \mathrm{s} 11606-022-07406-7$

(c) The Author(s) under exclusive licence to Society of General Internal Medicine 2022

\section{BACKGROUND}

Racial and ethnic minorities have disproportionately experienced COVID-19 cases and deaths, ${ }^{1}$ as well as excess deaths due to other causes, ${ }^{2}$ during the pandemic. There is growing concern that indirect effects of the pandemic, such as reduced access to care, may also be falling more heavily on people of color. Early pandemic data showed widespread delays or avoidance of medical care due to COVID-19 concerns, ${ }^{3}$ but it is unknown how these patterns have changed over time, particularly among racial/ethnic minority groups who have disproportionately been affected by unemployment, loss of insurance coverage, housing instability, and food insecurity. Understanding racial and ethnic differences in delaying or not seeking medical care is critically important and may help inform strategies to mitigate collateral effects of the pandemic.

\section{OBJECTIVE}

To evaluate the proportion of US adults who delayed or did not get needed medical care because of the COVID-19 pandemic by race/ethnicity, and how differences across groups changed over the course of the pandemic.

\section{METHODS}

We used US Census Bureau Household Pulse Survey (HPS) data from April 23, 2020, to May 10, 2021. ${ }^{4}$ The HPS is a 20-min online survey that was conducted weekly (April 2020-July 2020) and biweekly (August 19, 2020-onward) during the pandemic by the National Center for Health Statistics, to rapidly gauge and monitor the impact of the pandemic.

In this serial, cross-sectional study, our primary outcome was the proportion of respondents who delayed/did not get needed medical care because of the COVID-19 pandemic in the 4 weeks preceding the survey date. We calculated the

Received November 29, 2021

Accepted January 5, 2022

Published online February 8, 2022 proportion of adults who experienced this outcome by race/ ethnicity across four consecutive time periods: wave 1 (initial surge of US cases; April 23, 2020-June 16, 2020), post-wave 1 (June 18, 2020-September 14, 2020), wave 2 (winter surge of cases; September 16, 2020-February 15, 2021), post-wave 2 (February 17, 2021-May 10, 2021). Next, we fit logistic regression models to estimate the likelihood of delaying/not getting needed medical care by race/ethnicity using the nonHispanic(NH) White group as a reference. Our model included age, race/ethnicity, time period (e.g., wave 1), and an interaction term between race/ethnicity and time period.

All estimates were weighted to be nationally representative. Replicate weights provided by HPS were included for variance estimation. Analyses were conducted in SAS, version 9.4 (SAS Institute). IRB was not required due to the use of publicly available, de-identified data.

\section{FINDINGS}

A total of 2,470,717 adults participated in the survey. Weighted baseline characteristics are shown in Table 1. The proportion of all adults delaying/not getting medical care due to the pandemic decreased from $38.7 \%$ to $19.1 \%(P<0.001)$ over the study period; these patterns were similar across racial/ethnic groups (Fig. 1).

During wave 1 , Asian (OR 0.74; 95\% CI 0.69-0.80; $P<0.001$ ), Hispanic (OR 0.89; 95\% CI 0.84-0.93; $P<0.001$ ), and non-Hispanic Black (NH-Black) (OR 0.94; $95 \%$ CI 0.900.99; $P=0.01$ ) adults were less likely to delay/not get medical care than NH-White adults. During the post-wave 1 period, patterns were similar across racial/ethnic groups (Hispanic [OR 0.99; 95\% CI 0.95-1.04; $P=0.77$ ], NH-Black [OR 0.99; 95\% CI 0.96-1.02; $P=0.57]$ ), except for Asian adults (OR $0.85 ; 95 \%$ CI $0.81-0.90 ; P<0.001)$, who were less likely to delay/not get medical care than NH-White adults. During wave 2, Hispanic (OR 1.25; 95\% CI 1.20-1.29; $P<0.001$ ) and NH-Black (OR $1.05 ; 95 \%$ CI $1.00-1.10 ; P=0.04$ ) adults were more likely to delay/not get medical care than NH-White adults. By the end of the study period (post-wave 2), Asian (OR 1.22; 95\% CI 1.16-1.28; $P<0.001$ ), Hispanic (OR 1.39; 95\% CI 1.34-1.45; $P<0.001$ ), and NH-Black (OR 1.17; 95\% CI $1.12-1.22 ; P<0.001)$ adults were more likely to delay/not get medical care than $\mathrm{NH}-$ White adults. 
Table 1 Characteristics of the Respondents in the Household Pulse Survey, April 23, 2020, to May 10, 2021

\begin{tabular}{|c|c|c|c|c|c|}
\hline \multirow[t]{2}{*}{ Characteristics $^{\mathbf{a}, \mathbf{b}}$} & \multicolumn{5}{|c|}{ Weighted \% of Respondents } \\
\hline & $\begin{array}{l}\text { All participants } \\
(n=2,470,717)\end{array}$ & $\begin{array}{l}\text { Wave } 1 \\
(n=612,425)\end{array}$ & $\begin{array}{l}\text { Post-wave } 1 \\
(n=694,959)\end{array}$ & $\begin{array}{l}\text { Wave } 2 \\
(n=782,755)\end{array}$ & $\begin{array}{l}\text { Post-wave } 2 \\
(n=380,578)\end{array}$ \\
\hline Age, mean (SD) & $48.9(17.3)$ & $49.1(17.0)$ & $48.9(17.3)$ & $48.9(17.3)$ & $48.5(17.4)$ \\
\hline Male & 48.4 & 48.4 & 48.4 & 48.4 & 48.4 \\
\hline \multicolumn{6}{|l|}{ Race and ethnicity } \\
\hline White & 62.7 & 62.9 & 62.6 & 62.6 & 62.4 \\
\hline Black & 11.5 & 11.4 & 11.7 & 11.3 & 11.5 \\
\hline Hispanic & 16.9 & 16.8 & 16.9 & 17.0 & 17.1 \\
\hline Asian & 5.1 & 5.2 & 4.9 & 5.2 & 5.4 \\
\hline Other & 3.8 & 3.8 & 3.9 & 3.8 & 3.6 \\
\hline Education, high school or less & 39.2 & 39.2 & 39.2 & 39.2 & 39.1 \\
\hline Health insurance, yes & 90.0 & 89.8 & 89.5 & 90.3 & 90.8 \\
\hline Delay or did not get medical care, yes & 34.8 & 40.5 & 39.1 & 32.0 & 23.8 \\
\hline
\end{tabular}

${ }^{a}$ Observations with missing values were excluded from the analysis.

${ }^{b}$ All characteristics presented are self-reported by survey respondents.

cOur primary outcomes were defined as the proportion of individuals who responded "Yes" to either one of the following two survey questions: (1) At any time in the last 4 weeks, did you delay getting medical care because of the coronavirus pandemic? (2) At any time in the last 4 weeks, did you need medical care for something other than coronavirus, but did not get it because of the coronavirus pandemic?

\section{DISCUSSION}

The proportion of US adults delaying or not receiving medical care due to COVID-19 has declined over the course of the pandemic, although rates remain high $(\approx 20 \%$ as of May 2021$)$. White adults were most likely to miss medical care at the start of the pandemic. However, as the pandemic went on, racial and ethnic minority groups (Hispanic, Black, and Asian adults) became the most likely to delay/not receive medical care, and continue to be the most impacted groups. These widening disparities in health care access may in part be due to economic loss, financial strain, and loss of insurance

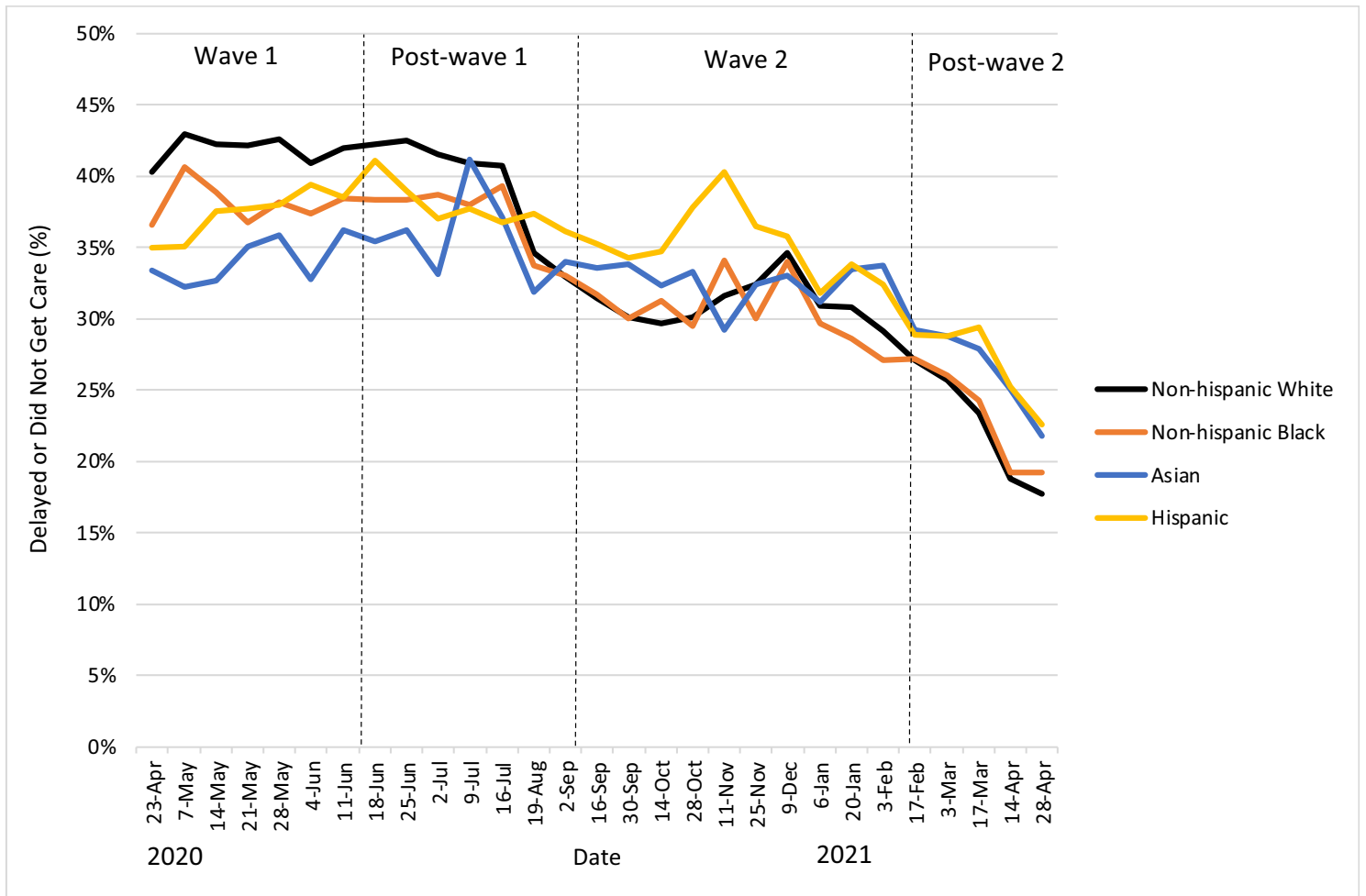

a Our primary outcomes were defined as the proportion of individuals who responded "Yes" to either one of the following two survey questions: (1) At any time in the last 4 weeks, did you delay getting medical care because of the coronavirus pandemic? (2) At any time in the last 4 weeks, did you need medical care for something other than coronavirus, but did not get it because of the coronavirus pandemic? 
coverage, all of which have fallen more heavily on Black and Hispanic families over the course of the pandemic, as well as differential uptake of vaccines. If these patterns continue, they may have important downstream repercussions for the health of these groups, and more broadly, health equity in the USA. Limitations of this study include potential selection bias due to non-response, although we used survey weights provided by HPS that are designed to account for variable response rates, and recall bias among respondents. ${ }^{5}$ Additionally, while we classified time periods according to when the initial surge and winter surge in cases occurred, there was variation in the timing of increases in cases across different parts of the country.

Nearly one in five US adults continue to delay/not receive medical care due to the pandemic. Public health and policy strategies are needed to increase access to medical care, particularly for racial/ethnic minorities.

\author{
Ahmed Ahmed, MPP, MSc ${ }^{1,2}$ \\ Yang Song, $\mathrm{MSc}^{1}$ \\ Rishi K. Wadhera, MD, MPP, MPhil ${ }^{1,2}$ \\ ${ }^{1}$ Richard A. and Susan F. Smith Center for Outcomes \\ Research in Cardiology, Beth Israel Deaconess \\ Medical Center, \\ Boston, MA, USA \\ ${ }^{2}$ Harvard Medical School, \\ Boston, MA, USA
}

Corresponding Author: Rishi K. Wadhera, MD, MPP, MPhil; Richard A. and Susan F. Smith Center for Outcomes Research in Cardiology, Beth Israel Deaconess Medical Center, Boston, MA, USA (e-mail: rwadhera@bidmc.harvard.edu).

\section{Declarations:}

Conflict of Interest: Dr. Wadhera receives research support from the National Heart, Lung, and Blood Institute (grant K23HL148525 and R01HL157530) at the National Institutes of Health. He serves as a consultant for Abbott outside the submitted work. All other authors have no disclosures.

\section{REFERENCES}

1. Wadhera RK, Wadhera P, Gaba P, Figueroa JF, Maddox KEJ, Yeh RW, et al. Variation in COVID-19 hospitalizations and deaths across New York City boroughs. JAMA [Internet]. 2020 Jun 2 [cited 2021 Oct 11];323(21):2192-5. Available from: https://jamanetwork.com/journals/jama/fullarticle/ 2765524

2. Wadhera RK, Figueroa JF, Rodriguez F, Liu M, Tian W, Kazi DS, et al Racial and ethnic disparities in heart and cerebrovascular disease deaths during the COVID-19 pandemic in the United States. Circulation [Internet]. 2021 Jun 15 [cited 2021 Sep 21];143:2346-54. Available from: https://www.ahajournals.org/doi/abs/10.1161/CIRCULATIONAHA. 121. 054378

3. Czeisler MÉ, Marynak K, Clarke K, Salah Z, Shakya I, Thierry J, et al. Delay or avoidance of medical care because of COVID-19related concerns - United States, June 2020. Morb Mortal Wkly Rep [Internet]. 2020 Sep 11 [cited 2021 Sep 2];69(36):1250-7. Available from: https://www.cdc.gov/mmwr/volumes/69/wr/ mm6936a4.htm

4. Bureau UC. Household Pulse Survey Data Tables [Internet]. [cited 2021 Sep 12]. Available from: https://www.census.gov/programs-surveys/ household-pulse-survey/data.html

5. Peterson S, Toribio N, Farber J, Hornick D. Nonresponse bias report for the 2020 Household Pulse Survey [Internet]. 2021 Mar [cited 2021 Sep 12]. Available from: https://www2.census.gov/programs-surveys/demo/technical-documentation/hhp/2020_HPS_NR_Bias_Report-final.pdf

Publisher's Note: Springer Nature remains neutral with regard to jurisdictional claims in published maps and institutional affiliations. 